\title{
POTENCIALIZA 3D: jogo para o ensino de formas geométricas básicas a discentes com Deficiência Intelectual
}

\author{
Francisco da C. Silva1, Fernando P. de Oliveira ${ }^{1}$, Elisiane M. Soares ${ }^{1}$, Erika P. M. \\ Ferreira $^{1}$, Thiago M. Pereira ${ }^{1}$, Emerson E. S. Moraes ${ }^{1}$, Gabriel S. Borges ${ }^{1}$, Jesiel B. \\ Santos ${ }^{1}$ \\ ${ }^{1}$ Departamento de Ensino - Instituto Federal de Educação, Ciência e Tecnologia \\ (IFMA - Campus Viana) \\ Rodovia MA 014 - KM 48 - Povoado São Pedro. CEP: 65.215-000 - Viana, MA - \\ Brasil \\ \{francisco.conceicao, fernando.pereira, elisiane.soares, erika \\ . ferreira, thiago.mourao\} @ifma.edu.br, \{e.emerson, gabrielborg \\ es\} @ acad.ifma.edu.br,jesiel.santos021099@gmail.com
}

\begin{abstract}
This study presents the Potencializa 3D game, developed in the light of the cultural-historical theory - CHT (VYGOTSKY, 1984), which purpose is to serve as a pedagogical instrument to the teacher in the teaching of basic mathematical concepts to students with Intellectual Disability (ID). The Potencializa 3D game proposes to work established correlations through basic geometric shapes, to better develop motor coordination, perception and notions of laterality. Its activities were developed based on the categories of mediation, zones of development and activity, of the CHT. The study arises from the need to provide to students with ID of the city of Viana-MA accessibility to technological resources in school routine, in a pedagogical way.
\end{abstract}

Resumo. Este trabalho apresenta o jogo Potencializa 3D, desenvolvido à luz da teoria histórico-cultural - thc (VYGOTSKY, 1984), cuja finalidade é servir como instrumento pedagógico ao professor no ensino de conceitos matemáticos básicos a discentes com Deficiência Intelectual (DI). O Potencializa 3D propõe trabalhar relações estabelecidas por meio de formas geométricas básicas, para melhor desenvolver a coordenação motora, percepção e noções de lateralidade. Suas atividades foram desenvolvidas com base nas categorias de mediação, zonas de desenvolvimento e atividade, da thc. O trabalho surge da necessidade de proporcionar aos discentes com DI da cidade de Viana-MA acessibilidade a recursos tecnológicos no cotidiano escolar, de forma pedagógica.

\section{Introdução}

A pessoa com Deficiência Intelectual (DI) se depara com obstáculos que afetam a apropriação de habilidades fundamentais à consolidação dos conteúdos escolares, tais como: percepção, raciocínio, memória, generalizações, atenção e motivação. Estas habilidades são necessárias para resolver problemas, entender ideias abstratas, manter relações sociais e praticar outras atividades do dia-a-dia (AAIDD, 2018; MALAQUIAS et al., 2013). Segundo o último Censo Demográfico (BRASIL, 2010), a população do Brasil corresponde a mais de 190 milhões de pessoas, sendo que 45 milhões $(24 \%)$ apresentam algum tipo de deficiência e desse total, mais de 2 milhões $(5,81 \%)$ correspondem à DI. 
Com a promulgação da Lei 9.394 de 1996, especialmente, ao tratar do paradigma inclusivo em seu capítulo $\mathrm{V}$, as escolas tem a oportunidade de construir caminhos que possibilitem o acesso e permanência de discentes com deficiência (BRASIL, 1996). Atualmente, a Lei 13.146 de 2015 reforça este paradigma, quando promove o uso de tecnologias assistivas e a superação de barreiras como da comunicação e informação, tecnológica, atitudinal, dentre outras, para promover acessibilidade às pessoas com necessidades especiais (BRASIL, 2015).

Ao se pensar nas possibilidades envolvidas no processo de ensino e aprendizagem de pessoas com DI, faz-se necessário a utilização de práticas pedagógicas que possibilitem acessibilidade a esse discente, para que sejam desenvolvidas suas habilidades cognitivas, motoras e construção criativa do conhecimento. Assim, os jogos são uma ferramenta interessante para tornar conteúdos maçantes em atividades envolventes e prazerosas, promovendo a motivação, disciplina e interesse do discente.

De acordo Vygotsky (1984), o desenvolvimento cultural do homem se dá a partir de um processo de aprendizado, que, necessariamente, é mediado. E o jogo tem a capacidade de proporcionar mediações entre o mundo imaginário e o real, pois, com ele é possível aprender a lidar com o mundo, recriando situações do dia-a-dia, possibilitando que o discente transfira o que aprendeu para outras situações de sua vida.

Uma aplicação muito interessante dos jogos está relacionada ao ensino da linguagem matemática, presente em todos os momentos da vida das pessoas, por exemplo, em habilidades como contar, comparar ou reconhecer formas de objetos. Para discentes com DI, porém, se apropriar deste conhecimento não é tão trivial, no entanto, os jogos podem potencializar o aprendizado e maior autonomia nestes discentes.

Neste sentido, o presente trabalho apresenta o jogo educacional Potencializa 3D, desenvolvido a partir de sua versão 2D (SILVA et. al. 2014), cujo objetivo é contribuir com o processo de ensino e aprendizagem de discentes com DI, da cidade de Viana-MA, abordando conteúdos iniciais da Matemática, por meio de figuras geométricas, tendo em vista os pressupostos teóricos da teoria histórico-cultural (thc). Para isso, foi realizado um estudo visando ampliar a compreensão acerca do desenvolvimento humano do discente com DI numa perspectiva histórico-cultural, investigado e definido técnicas e ferramentas de desenvolvimento de jogos digitais 3D; foram analisadas as atividades existentes e propostas novas atualizações para a versão 3D do jogo; por fim, foram implementadas as novas funcionalidades.

O artigo está organizado conforme segue: na seção 2 , é feita uma revisão de literatura, abordando o desenvolvimento humano do discente com DI à luz da thc. Na seção 3, são apresentados alguns trabalhos relacionados. A seção 4 apresenta o jogo Potencializa 3D. Na seção 5 temos as considerações finais e perspectivas de trabalhos futuros. Na seção 6, os agradecimentos e, por fim, as referências.

\section{O desenvolvimento humano do discente com DI numa perspectiva histórico-cultural}

A DI pode ser compreendida como limitações no funcionamento intelectual e no comportamento adaptativo, expresso em habilidades conceituais, sociais e práticas, com início anterior aos 18 anos (BRASIL, 1999). A pessoa com DI não tem alterada sua percepção enquanto ser humano e da realidade, sendo capaz de decidir o que é melhor para si (AAIDD, 2018). 
Assim, vemos a importância da compreensão desta deficiência, a fim de obtermos meios de proporcionar às pessoas nesta condição acesso à cultura historicamente acumulada, para que tenham possibilidade de apropriação das habilidades motoras e cognitivas, dentre elas, as referentes à matemática. Faz-se necessário criar situações motivadoras ao discente com DI para favorecer o avanço na sua compreensão, criandolhe conflitos cognitivos, desafiando-o a enfrentá-los, para que as atividades por ele realizadas tenham significado (VYGOTSKY, 1997).

Vygotsky ressalta que se faz necessário proporcionar uma educação social, que possibilite ao discente não adaptar-se à deficiência, mas vencê-la (VYGOTSKY, 1997). Desta forma, o presente trabalho aborda as categorias de mediação, zonas de desenvolvimento e atividade da thc (VYGOTSKY, 1984), que dão sustentação teórica ao jogo proposto, para promover o desenvolvimento das habilidades matemáticas em discentes com DI.

\subsection{Mediação}

A mediação está presente em todo processo de desenvolvimento de aprendizado, estando presente em toda atividade humana, definindo a relação do homem com o mundo de forma indireta (VYGOTSKI, 1997). Vygotsky (1988) entende a mediação como o processo que caracteriza a relação do homem com o mundo e com outros homens, sendo vista como central, pois é neste processo que as Funções Psicológicas Superiores (FPS) do homem se desenvolvem, tais como planejamento, memória voluntária e imaginação.

Luria (1992) aponta que, diante das características negativas de uma criança com deficiência, é necessário criar suas características positivas, por meio do uso de técnicas e habilidades culturais, que tornarão possível enfrentar uma tarefa inviável pelo uso de caminhos novos e diferentes. Desta forma, o autor afirma que o comportamento cultural apreendido pelo indivíduo irá se sobrepor à sua deficiência. Considerando o jogo proposto neste trabalho, o mesmo constitui-se como uma possibilidade inclusiva, pois, insere-se no contexto das técnicas e habilidades culturais proposto por Luria (1992), com objetivo de criar situações que conduzam ao desenvolvimento de habilidades matemáticas elementares, tais como a percepção, noções de lateralidade, concentração, dentre outras, a partir de relações estabelecidas por meio de formas geométricas básicas.

A mediação entre o conhecimento a ser apropriado a partir do jogo e o discente se dará através da interação mediante o uso de recursos audiovisuais, que orientarão o discente a mover-se pela tela para realizar as atividades propostas em forma de desafios. Esse caráter mediador inclui, ainda, os cenários das atividades, que representarão locais da cidade de Viana - MA, conhecidos pelo discente, e serão de natureza lúdica e pedagógica. Ressalta-se, ainda, a importância do professor como mediador do processo de aprendizagem do discente no uso do jogo, uma vez que o discente realizará atividades com níveis maiores de dificuldade, e, para isso, é necessário ter a ajuda de alguém mais experiente, que o estimule a fazer uso dos conhecimentos já apreendidos para a resolução de novos desafios.

\subsection{Zonas de Desenvolvimento}

O aprendizado na escola é um resultado desejável e a intervenção é um processo pedagógico privilegiado. Essa intervenção deve ser realizada conforme o nível de desenvolvimento em que o discente se encontra. 
Para Vygotsky (1984) existem duas zonas de desenvolvimento: a Real e a Proximal. A Zona de Desenvolvimento Real corresponde àquilo que o sujeito pode realizar de forma independente; é o nível de desenvolvimento das funções mentais, que resulta de ciclos de desenvolvimento já estabelecidos. A Zona de Desenvolvimento Proximal (ZDP) caracteriza tudo aquilo que o sujeito não é capaz de fazer de forma independente, ou ainda, o que o sujeito é capaz de fazer com ajuda de outro mais experiente, no qual a escola deveria atuar diretamente. Vygotsky (1984) entende a ZDP como potencializador da compreensão de uma série de pressupostos que dizem respeito ao processo de internalização, fundamentais na relação desenvolvimento - aprendizagem, no jogo, nas brincadeiras, e nas interações sociais, sendo que a aprendizagem acontece quando o ensino incide na ZDP.

As atividades do Potencializa 3D estão organizadas em forma de desafios, onde a atividade seguinte exigirá cada vez mais o empenho do discente em relação à anterior, isto é, buscará provocar conflitos cognitivos, desenvolver sua capacidade de concentração e raciocínio. Desta forma, serão criadas condições de promover no discente o pensamento abstrato, a partir de situações que retratem seu dia-a-dia.

A própria sequência de atividades visa possibilitar a ampliação de forma considerável da capacidade de concentração do discente, considerando que essas atividades devem começar tão fácil quanto seja necessário para que o discente com DI perceba que consegue executá-la, mas sempre com algum desafio. Desta forma, o aprendizado da atividade realizada possibilitará a realização de atividades mais desafiadoras, que atuarão na sua ZDP.

\subsection{Atividade}

A categoria Atividade é tratada na thc como sendo de grande importância para o processo de ensino e aprendizagem e, conforme Leontiev (2004), toda tarefa que a pessoa faz tem sempre um objetivo e um motivo, que darão sentido à tarefa. $\mathrm{O}$ objetivo é aquilo que deve ser alcançado no final da tarefa - seu resultado, que já é previsto como uma ideia, antes do início da ação. O motivo é a necessidade que leva a pessoa a agir. O sentido é dado pela relação entre o motivo e o objetivo - ou resultado - previsto para a tarefa.

O homem se apropria dos conteúdos da cultura humana, historicamente acumulada, por meio das atividades que realiza, transformando esses conteúdos em novos produtos culturais, desenvolvendo, assim, as FPS de sua conduta (LEONTIEV, 2004; VYGOTSKY, 1988). As atividades do jogo Potencializa 3D, através do seu caráter lúdico e desafiador, visam proporcionar ao discente interação com o jogo em um ambiente 3D, com efeitos sonoros e visuais atrativos, em cenários que representam locais de sua própria cidade (Viana - MA).

Algumas das atividades consistem no recolhimento de moedas enquanto o personagem se movimenta pelo cenário do jogo para formar as figuras geométricas, que, em seguida, devem ser reconhecidas pelo discente em uma lista de opções de figuras com formas diferentes. Assim, as atividades do jogo visam promover a motivação nos discentes, possibilitando que eles as realizem de forma prazerosa, onde os resultados decorrentes de suas ações coincidam com os objetivos anteriormente definidos para essas atividades. $\mathrm{O}$ jogo proposto neste trabalho, aliado à intervenção pedagógica do professor, é um importante instrumento no processo de ensino e aprendizagem do discente com DI. 
IX Congresso Brasileiro de Informática na Educação (CBIE 2020)

Anais do XXXI Simpósio Brasileiro de Informática na Educação (SBIE 2020)

\subsection{Ensino de Matemática para discentes com DI}

A Matemática é uma habilidade útil e necessária que é utilizada nas mais diversas atividades cotidianas e não cotidianas. No entanto, o discente com DI se depara com obstáculos no aprendizado desta disciplina, pois tem dificuldade em construir relações lógicas, necessárias para se apropriar de elementos fundamentais para a apreensão de conceitos matemáticos como, por exemplo, a abstração e a generalização.

Os discentes com DI são capazes de aprender e conseguem transferir o aprendizado para outras situações (ROSSIT, 2004; AAIDD, 2018) e sua aprendizagem é consequência do seu grau de motivação, sendo necessário definir estratégias pedagógicas bem estruturadas para o desenvolvimento da autonomia e constituição de sua autoimagem. O uso de jogos virtuais no ambiente escolar pode potencializar o ensino, em especial, da Matemática, tendo em vista a possibilidade de motivação quanto à perspectiva de aprendizagem. Para isso é importante que o professor explore dinâmicas diversificadas de ensino, através do uso de jogos eletrônicos educacionais.

\section{Trabalhos relacionados}

Esta seção descreve alguns trabalhos relacionados ao propósito desta pesquisa e que são de grande relevância para proporcionar acessibilidade a discentes com DI.

Medeiros et. al. (2019) apresentam uma sequência didática com o software SEBRAN ABC, software que conjuga atividades de língua portuguesa e matemática, que usam jogos para desenvolver o raciocínio lógico-matemático, a memória, a atenção e a concentração. Partem do pressuposto de que o uso de softwares educativos pode contribuir de modo significativo para o desenvolvimento de habilidades e competências nos discentes, especialmente os que apresentam alguma deficiência, e concluem que com a utilização destes jogos divertidos, os discentes podem ampliar as habilidades da Matemática e Língua Portuguesa.

O software VirtualMat é um ambiente virtual desenvolvido por Malaquias et al. (2013) que utiliza Realidade Virtual (RV) para trabalhar as principais características e limitações dos discentes com DI, especialmente as que interferem diretamente na construção do pensamento lógico-matemático. Conforme os autores, os resultados apontam que o software contribui significativamente para o desenvolvimento do pensamento lógico-abstrato dos discentes com DI. O software utiliza situações comuns da vida diária, oferecendo ao discente a possibilidade de desenvolver o pensamento lógico-matemático por meio da ludicidade.

Galvão et. al (2018) desenvolveram um aplicativo móvel com o objetivo de fornecer uma possibilidade adicional de entretenimento e lazer a discentes com DI, usando da ludicidade e aliando conceitos relacionados à capacitação destas pessoas para o trabalho, auxiliando-as em atividades rotineiras, simples e importantes, como, por exemplo: saudar as pessoas da maneira correta, consultar o calendário e relógio para aumentar a sua noção de tempo. Os autores relatam que o aplicativo obteve êxito em relação aos objetivos traçados, oferecendo, assim, uma possibilidade de lazer e entretenimento para pessoas com DI.

Nesse sentido, este trabalho apresenta a concepção de um software educacional, o jogo Potencializa 3D, para o ensino das habilidades matemáticas iniciais em discentes com DI, baseado nos pressupostos teóricos da thc. Suas atividades foram criadas em 
caráter de desafios a partir de uma perspectiva diferenciada dos softwares SEBRAN ABC e VirtualMat, tendo em vista que o jogo trabalha as relações estabelecidas por meio de formas geométricas básicas, visando potencializar a ampliação da coordenação motora, percepção e noções de lateralidade.

\section{O jogo Potencializa 3D}

Foi realizado o estudo da DI, na perspectiva da thc, para melhor compreensão das implicações decorrentes desta deficiência no processo de ensino e aprendizagem da linguagem matemática para discentes com DI. Em seguida, partindo da versão $2 \mathrm{D}$ do jogo, passou-se ao desenvolvimento das atualizações definidas: mudança da perspectiva 2D para a 3D, atualizações nas atividades existentes e criação de novas atividades.

\subsection{Desenvolvimento do jogo}

Os personagens, cenários e demais objetos foram criados manualmente, ou obtidos por fotos de espaços da cidade de Viana - MA, vetorizados através do software Corel Draw e modelados em 3D através do software Blender 3D. Para tratar da engenharia de manipulação, simulação, rastreamento de movimento e lógica do jogo, fezse uso da engine Unity. O Sony Vegas Pro foi utilizado para a criação da cutscene (presente na opção Assistir Trailer do jogo, é uma história baseada em ações, cujo objetivo é melhor contextualizar a narrativa de um jogo, facilitando sua compreensão e jogabilidade), através da técnica de animação 2D Stop Motion.

A implementação do jogo considerou aspectos pedagógicos direcionados ao público-alvo do trabalho e consistiu, principalmente, das seguintes criações:

- 2 personagens: um do sexo masculino e outro, do feminino;

- Animações: inclusão de movimentos de cabeça, braços e pernas, para dar um aspecto mais realista ao jogador;

- Trilhas sonoras e narrações em texto e áudios gravados com vozes naturais;

- 2 cenários: Praça da Bíblia e Praça da Matriz;

- Mapa de orientação: exibido na tela para que o jogador veja a disposição do personagem e a figura que está sendo criada no cenário;

- 3 atividades: apresentadas nas subseções 4.2.1, 4.2.2 e 4.2.3.

\subsection{Atividades pedagógicas}

A tela inicial do jogo contém as opções de atividades (ver figura 1): Treinar Movimentos e Iniciar Jogo (com as atividades Reconhecer Formas Geométricas e Criar Bandeiras).

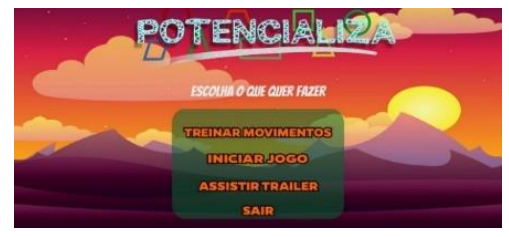

(a)

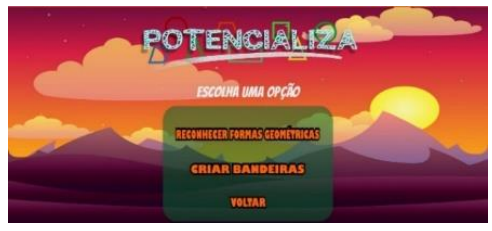

(b)

Figura 1. Telas de seleção de atividades: (a) Treinar Movimentos; (b) Formas Geométricas e Bandeiras.

A mediação ao discente nas atividades é realizada pelo jogo, por meio de orientações em áudio e texto, e pelo professor, através de suas intervenções pedagógicas. 
Para realizar uma atividade, o discente selecionará um dos cenários disponíveis (ver Figura 2a), e na tela seguinte, definirá o personagem que deseja utilizar para representálo no jogo (ver Figura 2b).

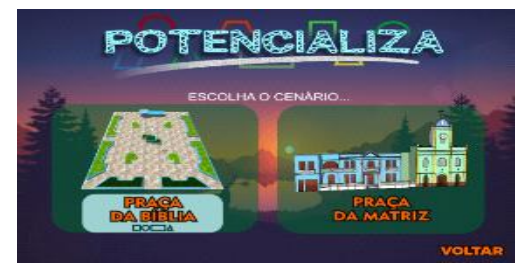

(a)

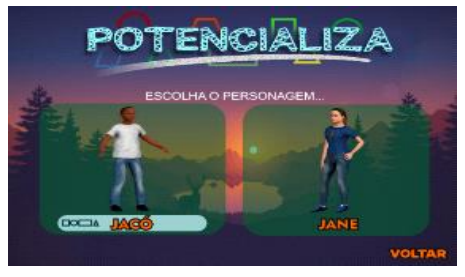

(b)

Figura 2. Telas de seleção: (a) Cenários; (b) Personagens.

Durante uma atividade, o discente será orientado pelo jogo a não colidir com os obstáculos do cenário, tais como muretas, árvores e postes, bem como do porquê de evitar estas colisões. Assim, dá-se uma interpretação ao erro do discente como parte do processo de aprendizagem, onde o erro é explorado e utilizado de maneira a gerar novos conhecimentos e investigações, num processo de trocas e produção do saber, promovendo a oportunidade de descobrir como esse discente organiza seu pensamento.

\subsubsection{Atividade Treinar Movimentos}

Esta atividade consiste na movimentação do personagem para coletar moedas pelo cenário. Serão trabalhadas habilidades como a percepção, noções de lateralidade e coordenação motora, para o discente se apropriar desse conhecimento, que atuará, assim, em sua ZDP, possibilitando realizar com êxito atividades mais desafiadoras no jogo.

Conforme Rossit (2004), discentes com DI apresentam dificuldade para compreender conhecimentos mais básicos, tais como, perceber alterações no ambiente (percepção), saber identificar o que é direita e esquerda (noções de lateralidade), bem como ter segurança no manuseio de objetos (coordenação motora). Assim, esta atividade é uma forma apropriada de se trabalhar a coordenação motora e habilidades matemáticas básicas (percepção e noções de lateralidade) no discente.

\subsubsection{Atividade Reconhecer Formas Geométricas}

Esta atividade consiste na definição das formas geométricas, onde o discente tem como tarefa coletar as diversas moedas espalhadas pelo cenário para formar a figura geométrica trabalhada naquele momento.

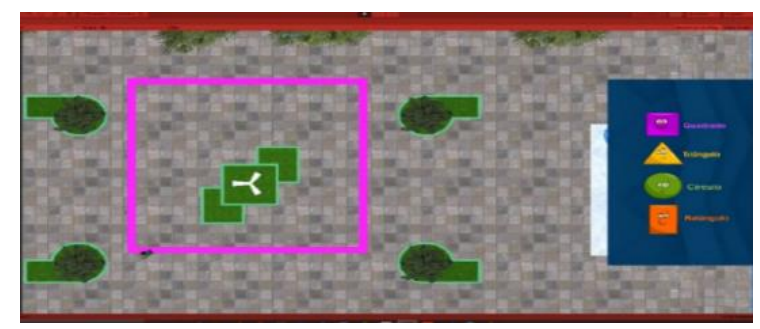

(a)

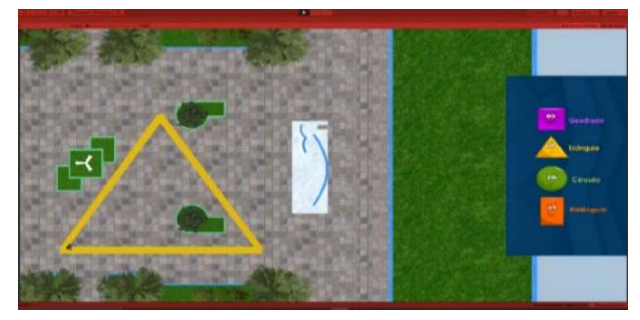

(b)

Figura 3. Figuras geométricas formadas pelo discente: (a) Quadrado; (b) Triângulo.

Ao completar a coleta das moedas, o discente terá formado uma figura geométrica, que será sinalizada com efeitos visuais, em uma perspectiva 2D. Neste momento, o 
discente será orientado por comando de voz e texto a identificar a figura formada, dentre várias outras opções disponíveis que surgirão na tela (ver figura 3).

Caso tenha êxito, serão dadas felicitações ao discente e uma nova atividade será gerada e novas moedas serão dispostas no cenário para serem recolhidas, formando a próxima figura geométrica. Caso o discente não identifique a figura formada, uma mensagem de áudio sobre o erro o conduzirá a uma reflexão a respeito da figura que o mesmo formou, permitindo que possa optar novamente pela figura geométrica correspondente. A mediação do professor neste momento é fundamental para criar situações que levem o discente a descobrir a figura formada, bem como reconhecê-la em situações do mundo real.

\subsubsection{Atividade Criar Bandeiras}

Esta atividade tem como objetivo fazer uso de figuras geométricas trabalhadas em momentos anteriores no jogo para a formação de um painel com bandeiras como a do Brasil e da cidade de Viana-MA. No cenário serão dispostas figuras em cores e formatos diversos e o discente terá que selecionar somente as figuras que completam corretamente a bandeira a ser montada no momento.

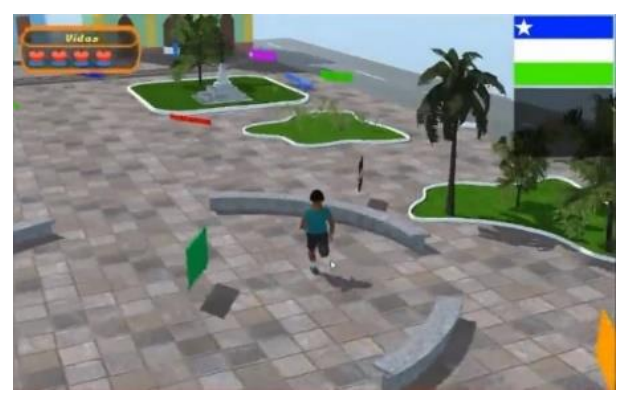

Figura 4. Formando a bandeira da cidade de Viana-MA.

As figuras 4 e 5 apresentam a realização da atividade, no momento em que está sendo formada a bandeira da cidade de Viana. Ao iniciar a atividade, o jogo orientará o discente sobre o objetivo da mesma, com a seguinte mensagem em áudio e texto: Várias figuras estão espalhadas pelo mapa da Praça da Matriz. Sua missão será coletar as figuras que formam a bandeira da Cidade de Viana. Boa Sorte!

Quando o discente coletar a última figura que compõe a bandeira, o jogo dará as congratulações ao jogador com a seguinte mensagem em áudio e texto: Parabéns! Você formou a bandeira da nossa querida cidade de Viana!!!

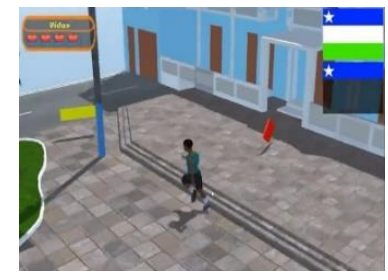

(a)

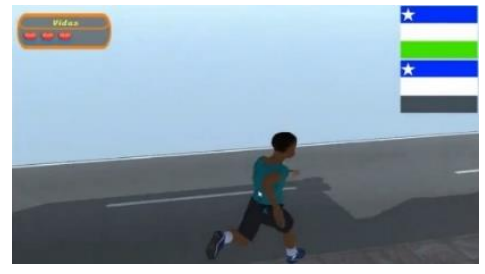

(b)

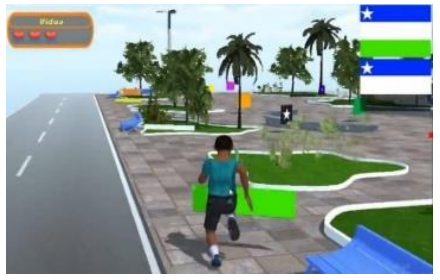

(c)

Figura 5. Formando a bandeira de Viana: (a) Inserindo a faixa azul; (b) Inserindo a faixa branca; (c) inserindo a faixa verde.

Após a formação de uma bandeira, será liberada outra atividade para que o discente faça o reconhecimento e coleta das formas geométricas que comporão nova bandeira, como a do Brasil (ver figura 6). 


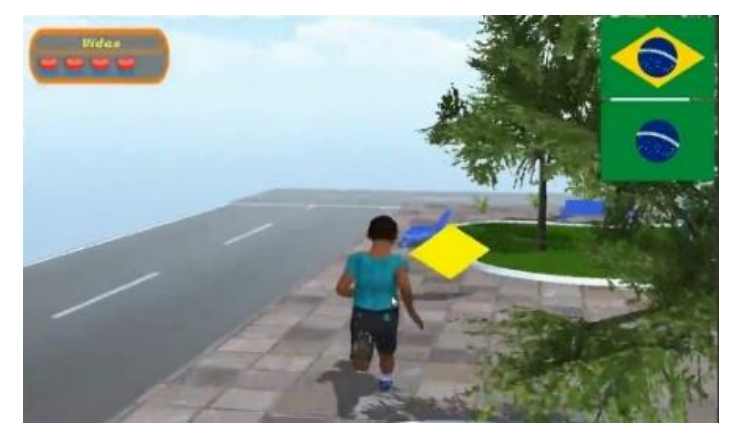

Figura 6. Formando a bandeira do Brasil.

A atividade de criação de bandeiras apresenta um nível maior de dificuldade, mas, a partir das atividades anteriores, em que foram trabalhadas a identificação destas formas geométricas, bem como, com o auxílio do professor, o discente terá todas as potencialidades para alcançar êxito na resolução do desafio.

\section{Considerações Finais}

O jogo Potencializa 3D foi desenvolvido com base em algumas categorias da teoria de Vygotsky, a thc, como as zonas de desenvolvimento, em que o conhecimento apropriado em atividades anteriores dará suporte para a realização de atividades mais desafiadoras.

Para isso, a mediação do professor será de grande importância, pois ele poderá instigar os discentes a refletirem sobre suas ações, a questionarem o porquê delas, proporcionando, assim, situações de aprendizagem que lhes permitam a apropriação das habilidades trabalhadas, dando condições para que o discente com DI não se adapte à sua deficiência, mas que a vença (VYGOTSKY, 1997).

Em se tratando de trabalhos futuros, pretende-se realizar as seguintes ações:

- Implementar e/ou atualizar novos recursos no jogo;

- Aplicar o jogo em escolas que atendam discentes com DI;

- Realizar coleta de dados e avaliação sobre a aplicação do jogo;

- Confeccionar produções científicas dos resultados obtidos da aplicação e avaliação do jogo para efeitos de publicação.

O desenvolvimento de uma proposta pedagógica como o Potencializa 3D, cujas atividades refletem planejamento do que se deseja ensinar, demonstra respeito ao indivíduo que necessita desta aprendizagem. Com isso, oportunidades de aprendizagens significativas são dadas para que o discente se torne um ser capaz, sendo sujeito de sua história, tendo as potencialidades para alcançar seu desenvolvimento cultural e humano.

\section{Agradecimentos}

Este trabalho foi financiado pela Pró-Reitoria de Pesquisa, Pós-Graduação e Inovação (PPGI) do Instituto Federal de Educação, Ciência e Tecnologia do Maranhão (IFMA), por meio do Programa de Bolsa Institucional de Desenvolvimento da Pesquisa (PIBIC), Edital Fábrica de Jogos, com execução no Campus Viana, deste instituto.

\section{Referências}

AAIDD (2018) American Association on Intellectual and Developmental Disabilities, 8403 Colesville Road, Suite 900 Silver Spring, MD 20910, Washington, D.C. 
Disponível em: https://www.aaidd.org/intellectual-disability/definition/faqs-onintellectual-disability. Acesso em: 18 de jun. 2020.

BRASIL. Lei ${ }^{\circ}$. 9.394, de 20 de dezembro de 1996. Lei de Diretrizes e Bases da Educação Nacional. Brasília, DF.

Decreto $\mathrm{n}^{\circ} 3.298$, de 20 de dezembro de 1999. Regulamenta a Lei $\mathrm{n}^{\circ} 7.853$, de 24 de outubro de 1989, dispõe sobre a Política Nacional para a Integração da Pessoa Portadora de Deficiência, consolida as normas de proteção, e dá outras providências. Disponível em: http://www.planalto.gov.br/ccivil_03/decreto/d3298.htm. Acesso em: 27 jun. 2020.

Instituto Brasileiro de Geografia e Estatística - IBGE. Censo 2010. Brasília, DF. Disponível em: https://censo2010.ibge.gov.br/. Acesso em: 18 de jun. 2020.

Lei n. 13.146, de 6 de jul. de 2015. Lei Brasileira de Inclusão da Pessoa com Deficiência. Disponível em: http://www.planalto.gov.br/ccivil_03/_Ato20152018/2015/Lei/L13146.htm. Acesso em: 27 jun 2020.

Galvão, L. R. et al. (2018). Desenvolvimento de um Aplicativo Lúdico para Pessoas com Deficiência Intelectual. In: XXIX Simpósio Brasileiro de Informática na Educação (SBIE). Disponível em: https://www.br-ie.org/pub/index.php/sbie/article/view/8048. Acesso em: 20 jun 2020.

Leontiev, A. N. (2004). O desenvolvimento do psiquismo. 2. ed. São Paulo: Centauro.

Luria, A.R. (1992). A Construção da Mente. São Paulo: Ícone.

Malaquias, F. F. O. et al. (2013). VirtualMat: A serious games to teach logicalmathematical concepts for students with intellectual disability. "Technology and disability", v. 25, n. 2.

Medeiros, S. A. et al. (2019). O Uso do Software SEBRAN ABC como Recurso Potencializador para o Desenvolvimento de Habilidades Matemáticas nas Pessoas com Deficiência Intelectual. In: IV Congresso sobre Tecnologias na Educação (Ctrl+E 2019). Disponível em: https://sol.sbc.org.br/index.php/ctrle/article/view/8901/8802. Acesso em: 20 jun 2020.

Rossit, R.A.S. Matemática para deficientes mentais: contribuições do paradigma de equivalência de estímulos para o desenvolvimento e avaliação de um currículo. 2004. Tese.

SILVA, F. C. et al. POTENCIALIZA: software para o ensino de atividades matemáticas iniciais em discentes com deficiência intelectual. In: NUEVAS IDEAS EN INFORMÁTICA EDUCATIVA TISE 2014. Fortaleza. 2014, Fortaleza.

Vygotsky, Lev Semenovich. (1984). A formação social da mente. São Paulo: Martins Fontes.

Aprendizagem e desenvolvimento intelectual na idade escolar. In: VIGOTSKII, L. S., LURIA, A. R. e LEONTIEV, A. N. Linguagem, desenvolvimento e aprendizagem. 4.ed. Tradução Maria da Penha Villalobos. São Paulo: Ícone: Universidade de São Paulo, 1988, p.103-118.

Fundamentos da Defectologia: Obras Completas. Tomo cinco. Cuba: Editorial Pueblo y Educación, 1997. 